\title{
Spontaneous Breakup of Extended Monodisperse Polymer Melts
}

\author{
Rasmussen, Henrik K.; Yu, Kaijia
}

Published in:

Physical Review Letters

Link to article, DOI:

10.1103/PhysRevLett.107.126001

Publication date:

2011

Document Version

Publisher's PDF, also known as Version of record

Link back to DTU Orbit

Citation (APA):

Rasmussen, H. K., \& Yu, K. (2011). Spontaneous Breakup of Extended Monodisperse Polymer Melts. Physical Review Letters, 107, [126001]. https://doi.org/10.1103/PhysRevLett.107.126001

\section{General rights}

Copyright and moral rights for the publications made accessible in the public portal are retained by the authors and/or other copyright owners and it is a condition of accessing publications that users recognise and abide by the legal requirements associated with these rights.

- Users may download and print one copy of any publication from the public portal for the purpose of private study or research.

- You may not further distribute the material or use it for any profit-making activity or commercial gain

- You may freely distribute the URL identifying the publication in the public portal

If you believe that this document breaches copyright please contact us providing details, and we will remove access to the work immediately and investigate your claim 


\title{
Spontaneous Breakup of Extended Monodisperse Polymer Melts
}

\author{
Henrik Koblitz Rasmussen and Kaijia Yu \\ Department of Mechanical Engineering, Technical University of Denmark, DK-2800 Kgs. Lyngby, Denmark
}

(Received 10 May 2011; published 13 September 2011)

\begin{abstract}
We apply continuum mechanical based, numerical modeling to study the dynamics of extended monodisperse polymer melts during the relaxation. The computations are within the ideas of the microstructural "interchain pressure" theory. The computations show a delayed necking resulting in a rupture, as a result of small initial sample imperfections. These ruptures agree with experimental observations.
\end{abstract}

DOI: 10.1103/PhysRevLett.107.126001

PACS numbers: 83.85.St, 83.10.Kn, 83.60.Uv

There have been remarkable advances in the understanding of polymer dynamics over the past few years [1-6], all based on tube models. This is a result of the appearance of new experimental techniques $[7,8]$. Still a lot of observations are unresolved. Particularly, the phenomenon of spontaneous ruptures in monodisperse polymer melts remains mysterious. The ruptures appear as developments of holes in thin films [9] or the delayed breakup of extended polymer melts $[10,11]$. These subjects have been discussed in a large number of scientific papers but are still unresolved. It has been argued that the spontaneous rupture in extended polymer melts is in contradiction with the idea behind the tube model [10]. However, it has been shown by numerical modeling that a tube model can show a delayed necking [12], which may be an initiation of a spontaneous breakup.

We will focus on the dynamics and breakup of monodisperse styrenebutadiene (SBR) polymer melts, as in the experimental studies by Wang and co-workers [10,11]. Wang and co-workers $[10,11]$ extended cylindrical-shaped SBR samples on a Sentmanat extension rheometer (SER) fixture [8]. At a given strain, the rotation of the cylinders was interrupted. As the stress relaxed, the dynamics and potential breakup of the samples were recorded. Our purpose is to study whether the published experiments can be explained within the framework of recent theoretical understandings of polymer dynamics.

We will use the recent constitutive equation by Wagner et al. $[2,13]$ based on the "interchain pressure" concept [1]. This approach can describe homogeneous flow behavior of monodisperse polymer melts accurately. The analytical (e.g., constitutive) model is

$$
\sigma_{i j}=\int_{-\infty}^{t} M\left(t-t^{\prime}\right) f\left(t, t^{\prime}\right)^{2} 5\left\langle\frac{E_{i n} u_{n} E_{j m} u_{m}}{|\mathbf{E} \mathbf{u}|^{2}}\right\rangle d t^{\prime},
$$

where

$$
\begin{gathered}
\frac{\partial}{\partial t} f\left(t, t^{\prime}\right)=f\left(t, t^{\prime}\right)\left[\frac{\partial}{\partial t}\langle\ln |\mathbf{E} \mathbf{u}|\rangle-\frac{1}{3 \tau_{R}} f\left(t, t^{\prime}\right)\left(f\left(t, t^{\prime}\right)^{3}-1\right)\right] \\
\text { and } f\left(t^{\prime}, t^{\prime}\right)=1 .
\end{gathered}
$$

$\tau_{R}$ is the Rouse time and $M\left(t-t^{\prime}\right)$ the memory function containing the linear dynamics of the polymer. $\sigma_{i j}$ are the components of the stress tensor. $f$ is referred to as the molecular stress function. The angular brackets denote an average over a unit sphere $\langle\cdots\rangle=1 /(4 \pi) \int_{|\mathbf{u}|=1} \ldots d \mathbf{u}$. u is a unit vector. The components of the displacement gradient tensor $\mathbf{E}$ are given by $E_{i j}\left(\boldsymbol{x}, t, t^{\prime}\right)=\partial x_{i} / \partial x_{j}^{\prime}$, $i=1,2,3$ and $j=1,2,3$. $\left(x_{1}^{\prime}, x_{2}^{\prime}, x_{3}^{\prime}\right)$ is the Cartesian coordinates of a given particle in the stress-free reference state (time $\left.t^{\prime}\right)$, displaced to coordinates $\left(x_{1}, x_{2}, x_{3}\right)$ in the current state (time $t$ ).

We will apply the method by Baumgaertel, Schausberger, and Winter [14] to describe the linear dynamics. The memory function is

$$
M\left(t-t^{\prime}\right)=\int_{0}^{\lambda_{\max }} n_{e} G_{N}^{0}\left(\frac{\lambda}{\lambda_{\max }}\right)^{n_{e}} \frac{e^{\left(t-t^{\prime}\right) / \lambda}}{\lambda^{2}} d \lambda,
$$

omitting the glassy part. $\lambda_{\max }$ is the maximal relaxation time in a continuous distribution of the time constant. Both $n_{e}$ and $G_{N}^{0}$ (the plateau modulus) have a unique value for each type of polymer. We will use the values obtained by Lyhne et al. [12], fitted for the $100 \mathrm{~K}$ SBR melt, for all the involved SBR melts. These are given in Table I. This table also contains the terminal relaxation time $\tau_{s}$ at room temperature for the different SBR melts, as determined by Wang and co-workers [10]. The terminal relaxation time is defined as the reciprocal of the crossover frequency, e.g., the angular frequency where the storage and loss moduli are equal in linear viscoelastic measurements. For all the SBR melts the ratio $\lambda_{\max } / \tau_{s}$ will be a fixed value as the number of entanglements $N$ in the melts are sufficiently high. Lyhne et al. determined one $\lambda_{\max }$ value for the $100 \mathrm{~K}$ SBR melt. Therefore, the remaining $\lambda_{\max }$ values can be calculated from the $\lambda_{\max } / \tau_{s}$ ratio.

TABLE I. SBR melt parameters at room temperature.

\begin{tabular}{cccrcc}
\hline \hline Name & $G_{N}^{0}$ & $n_{e}$ & \multicolumn{1}{c}{$\tau_{s}$} & $\lambda_{\max }$ & $\tau_{R}$ \\
\hline $100 \mathrm{~K}$ & $0.652 \mathrm{MPa}$ & 0.261 & $25 \mathrm{~s}$ & $57.95 \mathrm{~s}$ & $1.34 \mathrm{~s}$ \\
$170 \mathrm{~K}$ & $0.652 \mathrm{MPa}$ & 0.261 & $120 \mathrm{~s}$ & $278.2 \mathrm{~s}$ & $4.07 \mathrm{~s}$ \\
$250 \mathrm{~K}$ & $0.652 \mathrm{MPa}$ & 0.261 & $310 \mathrm{~s}$ & $718.6 \mathrm{~s}$ & $7.95 \mathrm{~s}$ \\
$500 \mathrm{~K}$ & $0.652 \mathrm{MPa}$ & 0.261 & $2100 \mathrm{~s}$ & $4868 \mathrm{~s}$ & $30.7 \mathrm{~s}$ \\
\hline \hline
\end{tabular}


The determination of the Rouse time highly depends on the used definition. In most definitions the Rouse time (relative to the terminal or the maximal relaxation time) is solely dependent on the number of entanglements of the polymer as $\tau_{R} / \tau_{s} \propto 1 / N$. The $100 \mathrm{~K} \mathrm{SBR}$ melt was reported [10] to have 24 entanglements. A value of $3 \tau_{R} / \lambda_{\max }=0.06962$ has been obtained for a polystyrene with 24 entanglements [7], based on startup of extension viscosities $\left(\bar{\eta}^{+}\right)$. The startup of the nominal extension viscosities $\left(\bar{\eta}_{N}^{+}\right)$for the $100 \mathrm{~K} \mathrm{SBR}$ melt is shown in Fig. 1 measured by [11] using a SER fixture [8]. The measured nominal viscosities agree well with the theoretical expectations, using the value of $3 \tau_{R} / \lambda_{\max }=0.06962$ in Eq. (2), as seen in Fig. 1.

Using the SER fixture, it is important to distinguish between the real strain $(\epsilon)$, as well as viscosities $\left(\bar{\eta}^{+}\right)$, and the nominal ones $\left(\epsilon_{N}\right.$ and $\bar{\eta}_{N}^{+}$, respectively). The real kinematic strain is defined as $\epsilon(t)=\ln [l(t) / l(0)]$, where $l(t)$ and $l(t=0)$ are the axial distances between two particles, theoretically separated by an infinitesimal distance, in the direction of the (axial) extension. $t$ is the time from the start of the extension at $t=0$. The real strain $(\epsilon)$ in the SER fixture is about $10 \%( \pm 3 \%)$ lower than the reported set strain $\left(\epsilon_{N}\right)$ [15], if a cylindrical sample with a radius of $R=1 \mathrm{~mm}$ is applied, as in $[10,11]$. It is important to notice that we use the relation $\epsilon=0.9 \epsilon_{N}$ in all our theoretical computations. For the corresponding strain rates $\dot{\epsilon}=0.9 \dot{\epsilon}_{N}$, where $\epsilon=\dot{\epsilon} t$. The nominal extension viscosities are defined as $\bar{\eta}_{N}^{+}=F /\left[\dot{\epsilon}_{N} A_{0} \exp \left(-\epsilon_{N}\right)\right]$, where $F$ is the axial (or extensional) force and $A_{0}$ the initial cross sectional area of the sample.

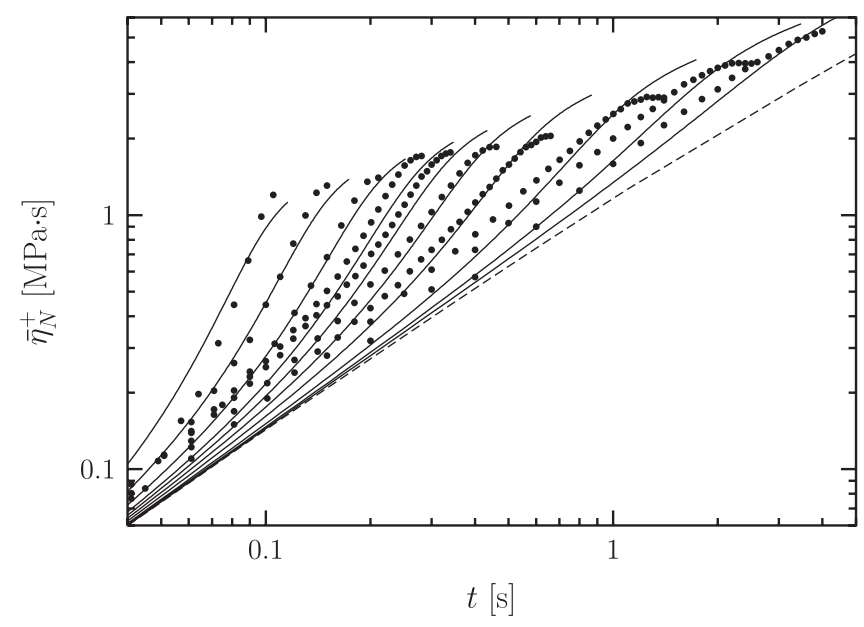

FIG. 1. The startup of nominal extension viscosities $\bar{\eta}_{N}^{+}$as function of the time $t$. $t$ is the time from the start of the extension (at $t=0$ ). The bullets $(\mathbf{)}$ ) are measurements from Fig. 7 in [11]. The set extensional rates $\dot{\epsilon}_{N}$ are $24,16,11,8,6.4,4.8,3.2,1.6$, 0.8 , and $0.4 \mathrm{~s}^{-1}$ from the left to the right curve. The solid lines are the corresponding theoretical prediction from Eqs. (1) and (2), with the use of the parameters in Table I for the $100 \mathrm{~K} \mathrm{SBR}$ melt. The dashed line is the linear viscoelastic envelope.
We apply the well-established relation $\tau_{s} \propto N^{3.4}$ [16], in the determination of the Rouse time for the remaining SBR melts. Removing the entanglement dependency using $\tau_{R} / \tau_{s} \propto 1 / N$, we obtain the relation $\tau_{R} \propto\left(\tau_{s}\right)^{2.4 / 3.4}$ or $\tau_{R} \propto\left(\tau_{\max }\right)^{2.4 / 3.4}$. Therefore, the remaining Rouse times $\left(\tau_{R}\right)$ will be calculated from this relation, and these values are given in Table I as well.

All the experiments by Wang and co-workers $[10,11]$ are performed on a SER fixture [8]. The SER fixture consists of two cylindrical drums. A circular cylinder with an initial radius of $R$ is attached onto these drums. $L_{0}$ is the length of the unsupported part of the sample in a SER fixture. Initially the sample is stress-free and at rest. At time $t=0$ the drums start the rotation. A constant set or nominal extension rate $\left(\dot{\epsilon}_{N}\right)$ is imposed on the sample, as the extension is achieved by counterrotating the drums with the same and constant angular rate. The rotation is halted at a set strain of $\epsilon_{N, 0}$ and real strain $\epsilon_{0}$, from whence the dynamics of the extended sample can be observed.

Computationally, this extension is modeled in axisymmetry with radial and axial coordinates $(r, z)$. We consider a cylinder of initial length $L(t=0)=L_{0} \exp \left(-\epsilon_{0}\right)$ and radius $R$. The cylinder is extended with a constant strain rate $(\dot{\boldsymbol{\epsilon}})$ as $L(t)=L_{0} \exp \left(\dot{\boldsymbol{\epsilon}} t-\epsilon_{0}\right)$ until $L=L_{0}$, where the length $L$ is kept constant. As $L_{0}=12.7 \mathrm{~mm}$ [8] the ratio $L_{0} / R=12.7$ in all computations. The length of the sample now corresponds to the length of the unsupported part of the sample in the SER fixture, and the dynamics of the sample can be followed computationally during its relaxation. This method ensures a reasonable numerical match of the real experimental conditions. Computationally we will use a geometry containing two planes of symmetry at the ends of the cylinder $(z= \pm L / 2)$ and at the center of the sample $(z=0)$. This allows a reduction in the computational domain to the area between $z=0$ and $z=L / 2$, where $z$ is the axial coordinate. This is the area contained between the dashed lines in Fig. 2. No surface tension, stress, or pressure is exerted on the free surface. We utilize the Lagrangian kinematic (e.g., particle) description from the used constitutive equation, where the numerical finite element method from Rasmussen [17] is applied. Notice that we apply the Currie approximation [18] in all our finite element computations, for the terms in the angular brackets in the constitutive equations (1) and (2).

The spontaneous breakup of extended monodisperse polymer melts would be considered an instability in a traditional continuum mechanical framework. It may be more descriptive to refer to it as a sensitivity to the initial conditions. Even minute differences from an ideal circular cylinder are bound to exist on the samples. Computationally the deviation from the ideal cylinder is positioned at the center of the sample, e.g., at $z=0$, although the observed break does not seem to occur at any particular place on the sample $[10,11]$. Here, the perturbation is realized as an axisymmetric sinusoidal-shaped suppression in the surface 


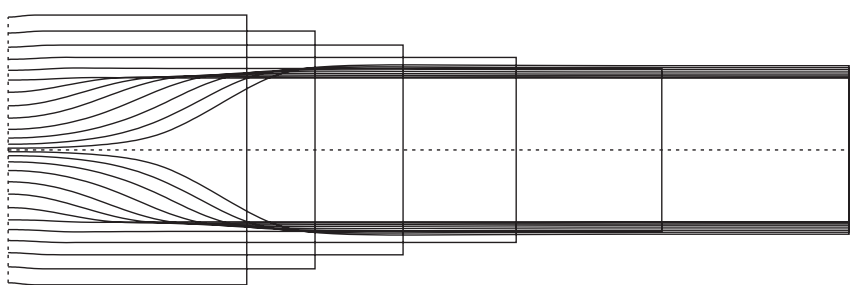

FIG. 2. The solid lines are the dynamic development of the boundary contours in the finite element modeling (of a $100 \mathrm{~K}$ SBR melt). $L_{0} / R=12.7$. The dashed lines are symmetry lines. The startup of flow, until a set strain of $\epsilon_{N, 0}$ (here $\epsilon_{N, 0}=1.4$ ) using a set extension rate of $\dot{\epsilon}_{N}$ (here $\dot{\epsilon}_{N}=9.6 \mathrm{~s}^{-1}$ ), is shown. It is following by the dynamic development of the boundaries, where the distance between the left and right boundaries is fixed. An initial perturbation of the cylindrical sample of $H / R=0.015$ and a width of $W / R=0.2$ is used in these finite element computations.

with height $H$ and a (total) width of $2 W$ (illustrated in Fig. 2) in the initial $(t=0)$ computational geometry. The evolution of an extension followed by relaxation is shown in Fig. 2. The sequence in Fig. 2 shows a numerically simulated sample extension, followed by the dynamics in the sample during the relaxation phase. A clear necking phase occurs during the relaxation.

A series of simulations has been performed for different imposed strains for the $100 \mathrm{~K}$ SBR melt. We have used a fixed initial perturbation height of $H / R=0.015$ and a width of $W / R=0.2$. The engineering stress $F / A_{0}$ is shown in Fig. 3 for the extension of the $100 \mathrm{~K}$ SBR melt.

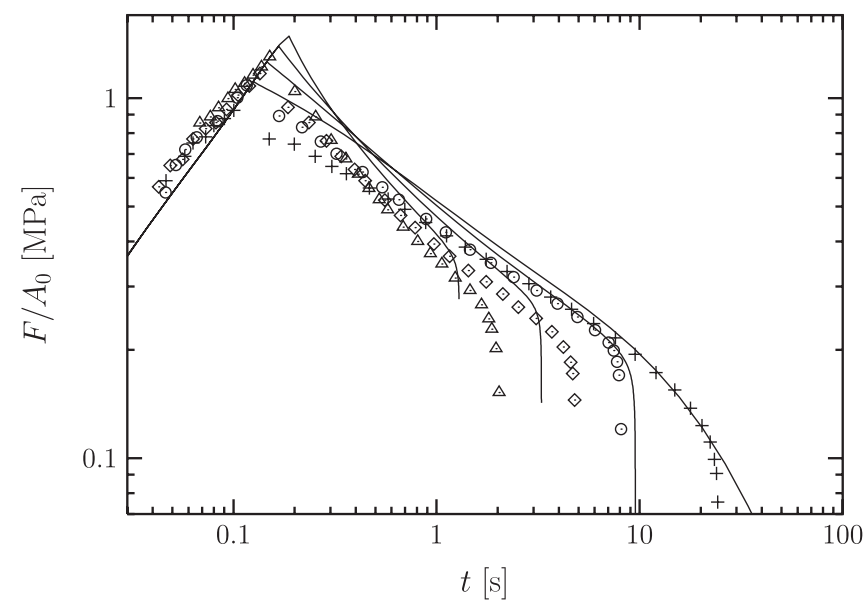

FIG. 3. The engineering stress $F / A_{0}$ as a function of the time from the initiation of the extension $(t=0)$ for the $100 \mathrm{~K} \mathrm{SBR}$ melt. The initial imposed set strain $\epsilon_{N, 0}$ is $1.8(\triangle), 1.6(\diamond)$, $1.4(\bigcirc)$, and $1.2(+)$, where the nominal extension rate $\dot{\epsilon}_{N}=9.6 \mathrm{~s}^{-1}$. The measurements are from Fig. 6 in [11]. The solid lines are the corresponding finite element computations to the symbols. They are based on Eqs. (1) and (2) (using the Currie approximation), with the parameters in Table I for the $100 \mathrm{~K}$ SBR melt. A fixed initial perturbation of the cylindrical sample height of $H / R=0.015$ and a width of $W / R=0.2$ is used in the finite element computations.
Our computations are shown together with the data from Wang and co-workers [11], originating from their Fig. 6. The necking occurs simultaneously with the sudden drop in the engineering stress and eventually evolves into a break. Although any initial imperfections of the samples will never be exactly the same in experiments, the computations show a remarkable agreement with the observations.

Similarly, a series of simulations (solid lines) has been performed for the different SBR melts, 170, 250, and $500 \mathrm{~K}$, for a fixed imposed strain. Here we have used a fixed initial perturbation height of $H / R=0.05$ and a width of $W / R=0.2$. Again, a remarkable agreement with the experimental data from Wang and co-workers [10] (originating from their Fig. 5) is observed in Fig. 4. For comparison we have also included the engineering stress for an ideal cylinder as the dashed lines. The sudden drop in the engineering stress is a result of the necking, initiating from the sample imperfection.

According to the hydrodynamic definition, a break is where a cross sectional area goes to zero in finite time. Numerical (finite element) modeling is not capable of finding this limit exactly. But in the computations in Figs. 3 and 4, and particularly in Fig. 2, the areas tend to go to zero in finite time for the computations showing a necking. To obtain an unambiguous measure of the occurrence of a break, we will define it as follows: the break happens if a linear extrapolation of the (smallest) cross sectional area of the sample $A$ versus the time $t$ will reach

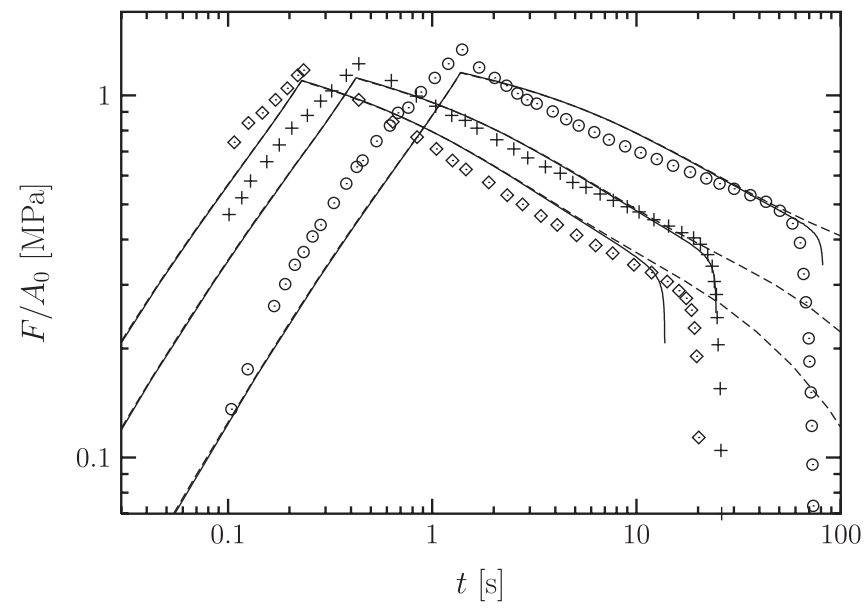

FIG. 4. The engineering stress $F / A_{0}$ as a function of the time from the initiation of the extension $(t=0)$. The $170(\diamond)$, $250(\bigcirc)$, and $500 \mathrm{~K}(+)$ SBR melts are represented in the left to the right curves, respectively. The initial imposed set strain rates $\dot{\epsilon}_{N}$ are $4.8(\diamond), 2.6(\bigcirc)$, and $0.8 \mathrm{~s}^{-1}(+)$ for the $170(\diamond), 250(\bigcirc)$, and $500 \mathrm{~K}(+)$ SBR melts, respectively. The initial imposed set strain $\epsilon_{N, 0}=1$.1. The solid lines are the corresponding finite element computations to the symbols. They are based on Eqs. (1) and (2) (using the Currie approximation), with the parameters in Table I for the respective SBR melts. A fixed initial perturbation of the cylindrical sample height of $H / R=0.05$ and a width of $W / R=0.2$ is used in the finite element computations. 


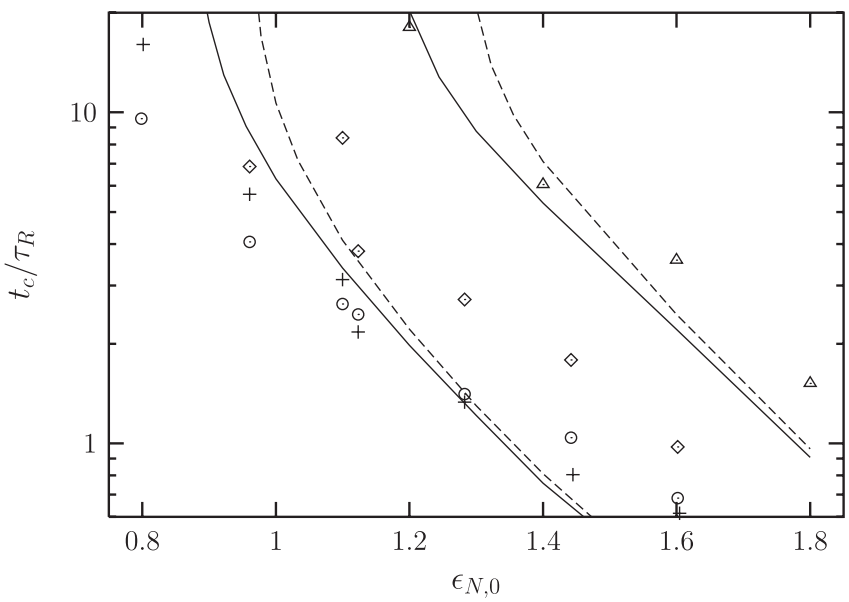

FIG. 5. Time to break $t_{c}$ relative to the Rouse time $\tau_{R}$ (from Table I) as function of the set strain where the rotation is halted $\epsilon_{N, 0}$. The measurements are from Fig. 5 in [10] and Fig. 6 in [11]. The initial imposed set strain rates $\dot{\epsilon}_{N}$ are $9.6(\triangle), 4.8(\diamond)$, $2.6(\bigcirc)$, and $0.8 \mathrm{~s}^{-1}(+)$ for the $100(\triangle), 170(\diamond), 250(\bigcirc)$, and $500 \mathrm{~K}(+)$ SBR melts, respectively. The lines are the finite element computations, based on Eqs. (1) and (2) (using the Currie approximation), with the parameters in Table I for the respective SBR melts. The dashed lines are finite element computations for the $100 \mathrm{~K} \mathrm{SBR}$ melt and the solid lines for the $250 \mathrm{~K} \mathrm{SBR}$ melt. A fixed initial perturbation of the cylindrical sample height of $H / R=0.05$ and a width of $W / R=0.2$ is used in the computations for the two lines to the left, where $H / R=0.015$ (the width is unchanged $W / R=0.2$ ) for the two lines to the right.

zero during a sufficiently small change in time. It may be written as the time $t_{c}=t$, where $A d t / d A<\delta t$, where $\delta$ is sufficiently small. Here we will use a $\delta$ value of 0.03 ; e.g., the actual break is expected to occur less than $3 \%$ later in time.

Figure 5 shows the time of the break $\left(t_{c}\right)$ relative to the Rouse time $\left(\tau_{R}\right)$ as a function of imposed set strain $\left(\dot{\epsilon}_{N, 0}\right)$. The symbols are the experimentally obtained break for all the involved SBR melts (from Fig. 5 and in [10], as well as Fig. 6 in [11]). The lines are the calculated break for the $100 \mathrm{~K} \mathrm{SBR}$ (dashed lines) and $250 \mathrm{~K} \mathrm{SBR}$ (solid lines) melts. The necking is a consequence of the relaxation of the molecular stress function, controlled by the Rouse time. Therefore, the necking and subsequent time of the break, relative to the Rouse time, are not very sensitive to the change of molecular weight. An exception occurs at low imposed strains. Here the transition from viscoelastic to viscous behavior, controlled by the relaxation time, stabilizes the sample. It is of interest to notice that the theoretical ideal lower limit for the occurrence of the necking, the elastic Considere criteria, is at a real strain of $\epsilon_{0}=0.86$ [19], corresponding to a set strain of $\dot{\epsilon}_{N, 0}=0.96$. Both the experiments and the computations show the break much earlier than this, as a consequence of the nonideal conditions.
Geometrically, we have only changed the sample height $H / R$, as the computations are relatively insensitive to the width $W / R$ of the disturbance. The computations show slopes similar to the experimentally observed breaks for the individual SBR melts, even though the consequence of changing the height of the disturbance is severe. In order to do a comparison between each of the individual experiments, information concerning the initial surface conditions needs to be monitored. This allows us to follow the temporal development of the surface from well-defined initial conditions both experimentally and with simulations.

Small initial deviations from a perfect cylindrical sample can result in delayed necking phenomena as observed during the relaxation of extended monodisperse polymer samples. These observed necking phenomena, resulting in a rupture, can be explained accurately within classical continuum mechanical instability theory. Here it is based on a continuum mechanical constitutive model for the fluid, within the ideas of the microstructural "interchain pressure" theory.

[1] G. Marrucci and G. Ianniruberto, Macromolecules 37, 3934 (2004)

[2] M. H. Wagner, S. Kheirandish, and O. Hassager, J. Rheol. 49, 1317 (2005).

[3] J. D. Schieber, D. M. Nair, and T. Kitkrailard, J. Rheol. 51, 1111 (2007).

[4] S. Dhole, A. Leygue, C. Bailly, and R. Keunings, J. NonNewtonian Fluid Mech. 161, 10 (2009).

[5] H. K. Rasmussen, A. L. Skov, J. K. Nielsen, and P. Laill, J. Rheol. 53, 401 (2009).

[6] D. Auhl, P. Chambon, T.C. B. McLeish, and D. J. Read, Phys. Rev. Lett. 103, 136001 (2009).

[7] A. Bach, K. Almdal, H. K. Rasmussen, and O. Hassager, Macromolecules 36, 5174 (2003).

[8] M. L. Sentmanat, Rheol. Acta 43, 657 (2004).

[9] G. Reiter, M. Hamieh, P. Damman, S. Sclavons, S. Gabriele, T. Vilmin, and E. Raphaë, Nature Mater. 4, 754 (2005).

[10] Y. Wang, P. Boukany, S.-Q. Wang, and X. Wang, Phys. Rev. Lett. 99, 237801 (2007).

[11] Y. Wang and S.-Q. Wang, J. Rheol. 52, 1275 (2008).

[12] A. Lyhne, H. K. Rasmussen, and O. Hassager, Phys. Rev. Lett. 102, 138301 (2009).

[13] M. H. Wagner and V.H. Rolón-Garrido, Rheol. Acta 49, 459 (2010).

[14] M. Baumgaertel, A. Schausberger, and H.H. Winter, Rheol. Acta 29, 400 (1990).

[15] K. Yu, H. K. Rasmussen, J. M. R. Marín, and O. Hassager, J. Rheol. 55, 571 (2011).

[16] S. T. Milner and T. C. B. McLeish, Phys. Rev. Lett. 81, 725 (1998).

[17] H. K. Rasmussen, J. Non-Newtonian Fluid Mech. 106, 107 (2002).

[18] P. K. Currie, J. Non-Newtonian Fluid Mech. 11, 53 (1982).

[19] G. H. McKinley and O. Hassager, J. Rheol. 43, 1195 (1999). 